\title{
INFLUÊNCIA DO TAMANHO DA SEMENTE NA EMERGÊNCIA DAS PLÂNTULAS DE LONGAN (Dimocarpos longan LOUR) ${ }^{1}$
}

\author{
INEZ VILAR DE MORAIS OLIVEIRA², RAQUEL SILVA COSTA³ ${ }^{3}$ RENATA APARECIDA DE ANDRADE ${ }^{4}$, \\ ANTONIO BALDO GERALDO MARTINS 5
}

\begin{abstract}
RESUMO - Objetivando-se avaliar a porcentagem de emergência de plântulas e o índice de velocidade de emergência (IVE) de sementes de longan (Dimocarpos longan Lour), o presente trabalho utilizou sementes obtidas de frutos maduros produzidos por plantas pertencentes ao Banco de Germoplasma do Departamento de Produção Vegetal da Faculdade de Ciências Agrárias e Veterinárias - UNESP, Câmpus de Jaboticabal- SP. Imediatamente após a extração, as sementes foram lavadas, secadas em papel jornal e semeadas em canteiros de areia. O delineamento experimental utilizado foi o inteiramente casualizado, com três tratamentos, caracterizados pelos tamanhos das sementes: grande, média e pequena, determinadas pela massa média de 50 sementes (em gramas), sendo: 89; 80 e 65g, respectivamente. Os dados de porcentagem de emergência de plântulas foram transformados em arc-sen $\sqrt{x / 100}$. As médias foram comparadas pelo teste de Tukey, a 5\% de probabilidade. Não foi observada diferença significativa para os parâmetros avaliados, sendo obtidos os valores de porcentagem de emergência de 97 a $91 \%$ e IVE de 0,651 a 0,680, demonstrando que, para a propagação sexuada desta espécie, o tamanho da semente não é fator a ser considerado.
\end{abstract}

Termos para indexação: longan; tamanho de sementes; emergência de plântulas

\section{INFLUENCE OF THE SIZE OF THE SEED ON EMERGENCE OF LONGAN (Dimocarpos longan LOUR) SEEDLING}

\begin{abstract}
With the objective to evaluate the percentage of seedlings emergence and the emergence velocity index (EVI) of longan (Dimocarpos longan Lour) seeds, was carried out the present research, using plants that came from the Germplasm Bank of the Department of Vegetable Production of the Faculdade de Ciências Agrárias e Veterinárias - UNESP - Campus of Jaboticabal, São Paulo state, Brazil. Immediately after the extraction, the seeds were washed, dryed and sowed in sand seed beds. The experimental delineation was entirely randomized, with three treatments, characterized by the size of the seeds: big, medium and small, determined by the medium weight of 50 seeds (in grams), being: 89 ; 80 and $65 \mathrm{~g}$ respectively. The values of percentage of emergence were transformed in arc-sen $\sqrt{x / 100}$. The averages were compared by the test of Tukey, with 5\% of probability. It was not observed significantly difference to the evaluated parameters, being obtained the values of the percentage of emergence of 97 to $91 \%$ and EVI of 0,651 to 0,680 , showing that the size of the seed is not a factor to be considered for this specie.
\end{abstract}

Index terms: longan; size of the seeds; seedling emergence.

A longan, longana ou olho-de-dragão (Dimocarpus longan Lour.), árvore frutífera da família Sapindaceae (assim como a lichia e o guaraná), é originária do sudeste asiático, mais precisamente da zona subtropical da China. E apesar de raramente conhecida, acha-se distribuída em todas as regiões subtropicais. Os frutos, apesar de assemelhados aos da lichieira, diferencia-se, quando maduros, pela coloração acastanhada e pelo sabor menos pronunciado (Ferrão, 1999).

De acordo com Carvalho \& Nakagawa (2000), em geral, as sementes de maior tamanho foram bem nutridas durante o seu desenvolvimento, possuindo embriões bem formados e com maior quantidade de substâncias de reserva, sendo, conseqüentemente, as mais vigorosas. A maior quantidade de reserva aumenta a probabilidade de sucesso no estabelecimento da plântula (Haig e Westoby, 1991), pois permite a sobrevivência por maior tempo em condições ambientais desfavoráveis. Popinigis (1985) afirma que o tamanho da semente, em muitas espécies, é indicativo de sua qualidade fisiológica. Assim, dentro do mesmo lote, as sementes pequenas apresentam menor emergência de plântulas e vigor do que as sementes de tamanho médio e grande. O objetivo do trabalho foi avaliar o efeito do tamanho da semente sobre a porcentagem e a velocidade de emergência de plântulas de longan.

O trabalho foi desenvolvido na Área Experimental de Produção de Mudas Frutíferas do Departamento de Produção Vegetal da Faculdade de Ciências Agrárias e Veterinárias - UNESP, Câmpus de Jaboticabal-SP.

As sementes foram extraídas manualmente de frutos maduros, provenientes de três matrizes pertencentes ao Banco de Germoplasma do Departamento de Produção Vegetal da FCAV, foram, após a extração dos frutos, lavadas em água corrente, secas à sombra por 24 horas e instaladas em canteiros de areia sob condições de ripado (50\% de luminosidade).

O delineamento experimental foi inteiramente casualizado, com 3 tratamentos, caracterizados pelos tamanhos das sementes: grande, médio e pequeno, determinados pela massa média de 50 sementes (em gramas), sendo 89; 80 e 65, respectivamente. Cada parcela foi composta por 10 sementes, com 10 repetições por tratamento.

A partir da semeadura em canteiros de areia sob condições de ripado (50\% de luminosidade), as plântulas emersas foram contadas semanalmente entre o início (14 dias após a instalação) da emergência e momento (42 dias após a instalação) de estabilização numérica das contagens. Os dados obtidos permitem calcular índice de velocidade de emergência (Maguire, 1962) e, ao mesmo tempo, a taxa (\%) de emergência resultante da totalização das contagens. Os dados de porcentagem de emergência de plântulas foram transformados em arc-sen $\sqrt{x / 100}$ para análise estatística. As médias foram comparadas pelo teste de Tukey, a $5 \%$ de probabilidade.

Pelos resultados referentes ao IVE e porcentagem de emergência (Tabela 1), verificou-se que não houve diferença na emergência das sementes em função do tamanho. Resultados similares foram encontrados por Ferreira \& Torres (2000) estudando a influência do tamanho das sementes de Acacia senegal (L.) na germinação e no vigor, os quais verificaram que a porcentagem e o índice de velocidade de emergência não foram afetados pelo tamanho das sementes. Em sementes de soja cv. IAC-2, separadas em mesa gravitacional e por

\footnotetext{
(Trabalho 177/2004). Recebido: 15/11/2004. Aceito para publicação: 19/04/2005.

${ }^{2}$ Eng. Agr., Msc, Aluna de Pós-graduação em Agronomia - Produção Vegetal - UNESP - FCAV - Depto de Produção Vegetal. Via de acesso Prof. Paulo Donato Castellane, s/n. Cep: 14884-900. Jaboticabal/SP. Tel/Fax: (16)32092668. e-mail: inezvilar@yahoo.com. Bolsista CAPES.

${ }^{3}$ Bióloga, Msc, Aluna de Pós-Graduação em Agronomia - Genética e Melhoramento de Plantas - UNESP - FCAV - Depto de Biologia Aplicada. Tel: (16)32092620. e-mail: rscosta@fcav.unesp.br. Bolsista CNPq.

${ }^{4}$ Eng. Agr., Msc., Aluna de Pós-graduação em Agronomia - Produção Vegetal - UNESP - FCAV - Depto de Produção Vegetal. e-mail: reandrad@ @cav.unesp.br. Bolsista CNPq.

${ }^{5}$ Eng. Agr., Prof. Doutor - UNESP - FCAV - Depto de Produção Vegetal. e-mail: baldo@ fcav.unesp.br
} 
diferentes peneiras, classificadas como sementes leve e pesada e por três tamanhos: pequena, média e grande, Feldmann \& Toledo (1979) observaram que tanto o peso quanto o tamanho não exerceram influência sobre a germinação, vigor e produção. Aguiar et al. (1979), analisando duas espécies de eucaliptos (Eucaliptus grandis e Eucaliptus urophyla), concluíram que a capacidade de germinação não foi afetada pelo tamanho das sementes.

TABELA 1 - Dados médios obtidos para os tratamentos nas determinações do índice de velocidade (IVE) e da taxa de emergência das plântulas.

\begin{tabular}{cccc}
\hline Tratamento & IVE & \multicolumn{2}{c}{ Taxa de emergência (\%) } \\
\cline { 3 - 4 } Tamanho & & $\begin{array}{c}\text { Dados } \\
\text { originais }\end{array}$ & $\begin{array}{c}\text { Dados } \\
\text { transformados }\end{array}$ \\
\hline semente (g) & & 97,00 & $84,25 \mathrm{a}$ \\
Mrande & $0,676 \mathrm{a}$ & 91,00 & $78,29 \mathrm{a}$ \\
Pequeno & $0,680 \mathrm{a}$ & 92,00 & $73,37 \mathrm{a}$ \\
\hline F & $0,651 \mathrm{a}$ & & $1,24^{\mathrm{ns}}$ \\
Dms (5\%) & $1,47^{\mathrm{ns}}$ & & 11,77 \\
Desv. Pad. (5\%) & 8,35 & & 10,61 \\
CV (\%) & 7,53 & & 13,27 \\
\hline
\end{tabular}

*Médias seguidas de mesma letra na coluna não diferem entre si pelo teste de Tukey, $(5 \%)$ de probabilidade.

Rego et al. (1991), em estudo com macadâmia (Macadamia integrifólia Maiden e Betche), evidenciaram que a porcentagem e o índice de velocidade de germinação não foram afetados pelo tamanho das sementes que, embora grandes e contendo aparentemente maior quantidade de reservas, não apresentaram o melhor desempenho germinativo. Entretanto, Malcolm et al. (2003), estudando o efeito do peso da semente na porcentagem de germinação de sementes de cinco cultivares de porta-enxertos para pêssego, observaram que as sementes mais pesadas tiveram maior porcentagem de germinação e germinaram mais rapidamente.

O início da emergência ocorreu na segunda semana (14 dias) após a instalação do experimento, com valores de emergência de 46; 51 e $58 \%$ para as sementes de tamanho pequeno, médio e grande, respectivamente. Houve aumento progressivo desses valores durante as demais semanas de avaliação, atingido máximo de $92 \%$ (tamanho pequeno), $91 \%$ (médio) e $97 \%$ (sementes de tamanho grande) na sexta semana, determinando então a duração do experimento.
Dessa forma, qualquer tamanho de semente pode ser utilizado tanto objetivando a propagação para fins de produção comercial como para melhoramento da espécie, tendo em vista os resultados obtidos.

As diferenças entre os tamanhos das sementes de longan (Dimocarpus longan Lour.) não interferem na emergência das plântulas. Agradecimento à CAPES, pela concessão de bolsa de estudo à Inez Vilar de Morais Oliveira.

\section{REFERÊNCIAS}

AGUIAR, I.B.; CARVALHO, N.M.; MAIMONI-RODELLA, R.C.S.; DAMASCENO, M.C.M. Influência do tamanho sobre a germinação e vigor de sementes de eucalipto. Revista Brasileira de Sementes, Brasília. v.1, n.1, p.53-58, 1979.

CARVALHO, N.M.; NAKAGAWA, J. Sementes: ciência tecnologia e produção. 4.Ed. Jaboticabal: FUNEP, 2000, 588p.

FELDMANN, R. de O.; TOLEDO, F. F. Influência do peso e do tamanho da semente sobre a germinação, vigor e a produção da soja (Glycine $\operatorname{Max}(\mathrm{L}$.) Merrill). In: CONGRESSO BRASILEIRO DE SEMENTES, Curitiba, 1979. Informativo Abrates. Brasília, 1979. p.28.

FERRÃO, J.E.M.; Fruticultura tropical: espécies com frutos comestíveis. Lisboa : Instituto de Investigação Científica Tropical, 1999. v.1, p. 524.

FERREIRA, M. G. R.; TORRES, S. B. Influência do tamanho das sementes na germinação e no vigor de plântulas de Acacia Senegal (L.) Willd. Revista Brasileira de Sementes, Brasília, v. 22, n. 1, p. 271-275, 2000.

HAIG, D.;WESTOBY, M. Seed size, pollination casts and angiosperm success. Evolutionary Ecology, London, v. 5, p. 231-247, 1991.

MAGUIRE, J. D. Speed of germination - aid in selection evolution for sudling emergence and vigor. Crop Science, v.2, n.2, p.176-177, 1962.

MALCOLM, P.J.; HOLFORD, P.; McGLASSON, W.B.; NEWMAN, S. Temperature and seed weight affect the germination of peach rootstock seeds and the growth of rootstock seedlings. In: Scientia Horticulturae, 98, p. 247-256, 2003.

POPINIGIS, F. Fisiologia da semente. AGIPLAN, Brasília, 1985. 289p.

REGO, F. A. O.; COSTA, M. M. N.; ABREU, S. M.; SILVA, A. Q.; SILVA, H. Influência do tamanho da semente e escarificação na germinação de macadâmia (Macadamia integrifólia). In: CONGRESSO BRASILEIRO DE SEMENTES, 7, Campo Grande, 1991, Resumos. Informativo ABRATES, Londrina, v. 1, n. 4, p.85, 1991. 